\title{
Evidence of genetic enrichment for exceptional survival using a family approach: the Leiden Longevity Study
}

\author{
Manja Schoenmaker ${ }^{1}$, Anton JM de Craen*, ${ }^{\star 1}$, Paul HEM de Meijer ${ }^{2}$, Marian Beekman ${ }^{3}$, \\ Gerard J Blauw ${ }^{1}$, P Eline Slagboom ${ }^{3}$ and Rudi GJ Westendorp ${ }^{1}$
}

\author{
${ }^{1}$ Department of Gerontology and Geriatrics, Leiden University Medical Center (LUMC), Leiden, The Netherlands; \\ ${ }^{2}$ Department of General Internal Medicine, Leiden University Medical Center (LUMC), Leiden, The Netherlands; \\ ${ }^{3}$ Section of Molecular Epidemiology, Leiden University Medical Center (LUMC), Leiden, The Netherlands
}

We conducted a sib pair study in very old subjects for the purpose of mapping longevity loci. In the present analysis, we explore whether our recruitment strategy has resulted in a population enriched for a heritable component for exceptional longevity. Our study includes families with at least two longliving siblings (men aged 89 years or above; women aged 91 years or above). Data were collected on date of birth and, if applicable, date of death of parents, brothers and sisters, offspring, and spouses of the long-living participants. Standardised mortality ratios (SMRs) compared with the general Dutch population, were calculated. The SMR for all siblings of the long-living participants was $0.66(95 \%$ $\mathrm{Cl} 0.60-0.73)$. A similar survival benefit was also observed in the parents ( $\mathrm{SMR}=0.76,95 \% \mathrm{Cl} 0.66-0.87$ ) and in the offspring of the long-living subjects $(\mathrm{SMR}=0.65,95 \% \mathrm{Cl} 0.51-0.80)$. The SMR of the spouses of the long-living subjects was $0.95(95 \% \mathrm{Cl} 0.82-1.12)$. The familial clustering of extended survival is unlikely to be caused by ascertainment bias, because in all analyses the long-living participants were excluded. Moreover, it is also unlikely to be caused by environmental factors, because the spouses of the long-living participants had a mortality risk comparable with the general Dutch population, whereas they share the same environment. We conclude that our sample is genetically enriched for extreme survival.

European Journal of Human Genetics (2006) 14, 79-84. doi:10.1038/sj.ejhg.5201508; published online 26 October 2005

Keywords: longevity; epidemiology; genetics

\section{Introduction}

Each species has its own characteristic lifespan. ${ }^{1}$ However, within a species, individual lifespans vary widely. This variation is in part the result of an individual ability to avoid or cope with internal and external damage, which has a strong genetic basis. ${ }^{2}$ For example, single

*Correspondence: $\operatorname{Dr}$ AJM de Craen, Department of Gerontology and Geriatrics, C-2-R Leiden University Medical Centre, PO Box 9600, 2300 RC Leiden, The Netherlands. Tel: + 3171526 6640; Fax: + 3171524 8159; E-mail: craen@lumc.nl

Received 13 April 2005; revised 6 July 2005; accepted 16 September 2005; published online 26 October 2005 point mutations in the more than 17000 genes of Caenorhabditis elegans can lower the rate of aging and lengthen lifespan up to nearly five times as long as the wild-type worms. ${ }^{3}$ In mice, a single point mutation in the p66shc gene delays the rate of aging and extends average lifespan by about $30 \%{ }^{4}$ These experimental data suggest that the majority of age-related changes are under coordinated genetic control. ${ }^{5}$

By definition, experimental research into aging has been performed in model organisms. However, several observational studies in humans have also explored the genetic component in susceptibility to death. During the last 
decade, a number of twin studies have shown that approximately $25 \%$ in the variation of human lifespan is explained by genetic factors. ${ }^{6,7}$ The remainder of the variation has to be explained by private environmental factors and gene-environment interaction. Moreover, recent studies have demonstrated a clustering of extreme longevity within families. ${ }^{8,9}$ Puca et al ${ }^{10}$ reported on the localisation of a longevity locus on chromosome 4 after a genome-wide scan in 308 individuals belonging to 137 long-living sibships. Additionally, Reed et al ${ }^{11}$ identified the same region as Puca on chromosome 4 in 95 healthy male twin pairs with a mean age of 74 years. Hence, it is likely that genetic factors play an important role in human longevity, but the exact pathways remain to be elucidated.

The aim of the Leiden Longevity study is to locate genetic loci explaining the interindividual differences in human longevity using an affected sib pair design. Therefore, we collected a large series of sib pairs aged 90 years and over. By collecting sib pairs instead of long-living singletons, we expected an enrichment of genetic factors contributing to longevity in this population, whereas the likelihood of having reached a long life because of exceptional environmental conditions or chance would be lower. Here we report whether our study of long-living sib pairs has indeed resulted in a population genetically enriched for longevity.

\section{Methods}

Families can participate in the Leiden Longevity Study if at least two siblings are long-living. There are four inclusion criteria for the long-living subjects: (1) men must be aged 89 years or above and women must be aged 91 years or above; (2) subjects must have at least one living brother or one living sister who fulfils the first criterion and is willing to participate; (3) the sib pairs have an identical mother and father; (4) the parents of the sibship are Dutch and Caucasian. The minimum age of 89 years for men and 91 years for women was chosen because the age of the participants needed to be extreme enough to be considered long-lived. In 2001, less than $0.5 \%$ of the Dutch population fulfilled these sex-specific criteria. We have not increased these age criteria any further because we then would not be able to recruit a series of 500 long-living sib pairs from a source population of about 16 million people. In accordance with the Declaration of Helsinki, we obtained informed consent from all participants prior to their entering the study. Good clinical practice guidelines were maintained. The study protocol was approved by the ethical committee of the Leiden University Medical Center before the start of the study.

In total, 500 sibships were collected to enable a genomewide scan for longevity. Long-living cousins are also asked to participate. In addition, the offspring of the long-living sib pairs and their partners were enrolled to enable future association studies. In these studies, the offspring of long-living subjects, who are assumed to have a higher susceptibility to become long-lived, will be the cases, and their partners, who are representatives of the general population, will be the controls. One of the advantages of using partners of offspring of long-living subjects as the control population is that they are likely to have the same age, socio-economic, and geographical background.

\section{Data collection}

All long-living subjects were visited at home, preferably in the company of a next-of-kin. During the visit the pedigrees were completed (see below) and a number of questionnaires were administered. Cognitive performance is assessed with the Mini-Mental State Examination (MMSE). ${ }^{12}$ We defined severe cognitive impairment as an MMSE-score below 19 points. In case of cognitive impairment, informed consent and assessment on competence and actual performances in activities of daily living (ADL) were obtained from a guardian. Disabilities in ADL are measured with the Barthel ADL Index ${ }^{13}$ and a modified Fillenbaum questionnaire. ${ }^{14}$ The sum score of the Barthel Index ranges from 0 (unable to perform any activity) to 20 (competent in all activities). The Fillenbaum questionnaire has a sum score that ranges from 0 (unable to perform any activity) to 14 (competent in all activities). The Cantril ladder, ${ }^{15}$ a visual analogue scale on perceived quality of life varying from 1 to 10 points, is administered to assess wellbeing.

Each participating family provides us with the genealogical information regarding the parents (ie P1), all siblings (ie F1), and the offspring (ie F2) of the long-living participants. With this genealogical data, three-generation pedigrees were constructed (see Figure 1). Whenever possible, this information was verified by passport, or by birth or marriage certificate. Moreover, all data were also verified with the personal record cards of the deceased family members in the national population registry located at the Central Bureau of Genealogy in The Hague, The Netherlands.

From the long-living subjects, their offspring, the partners of their offspring, and the long-living cousins, a venous blood sample was drawn for isolation of DNA, RNA, serum, and plasma. In case a participant prefered a mouth swab instead of a blood sample, a mouth swab was collected for DNA-extraction only. Moreover, place of birth of the parents of the partners of the offspring was recorded, to facilitate classification of partners as being 'Caucasian'.

\section{Statistical methods}

The statistical analysis proceeded in various stages. First, the mortality of various generations was compared 


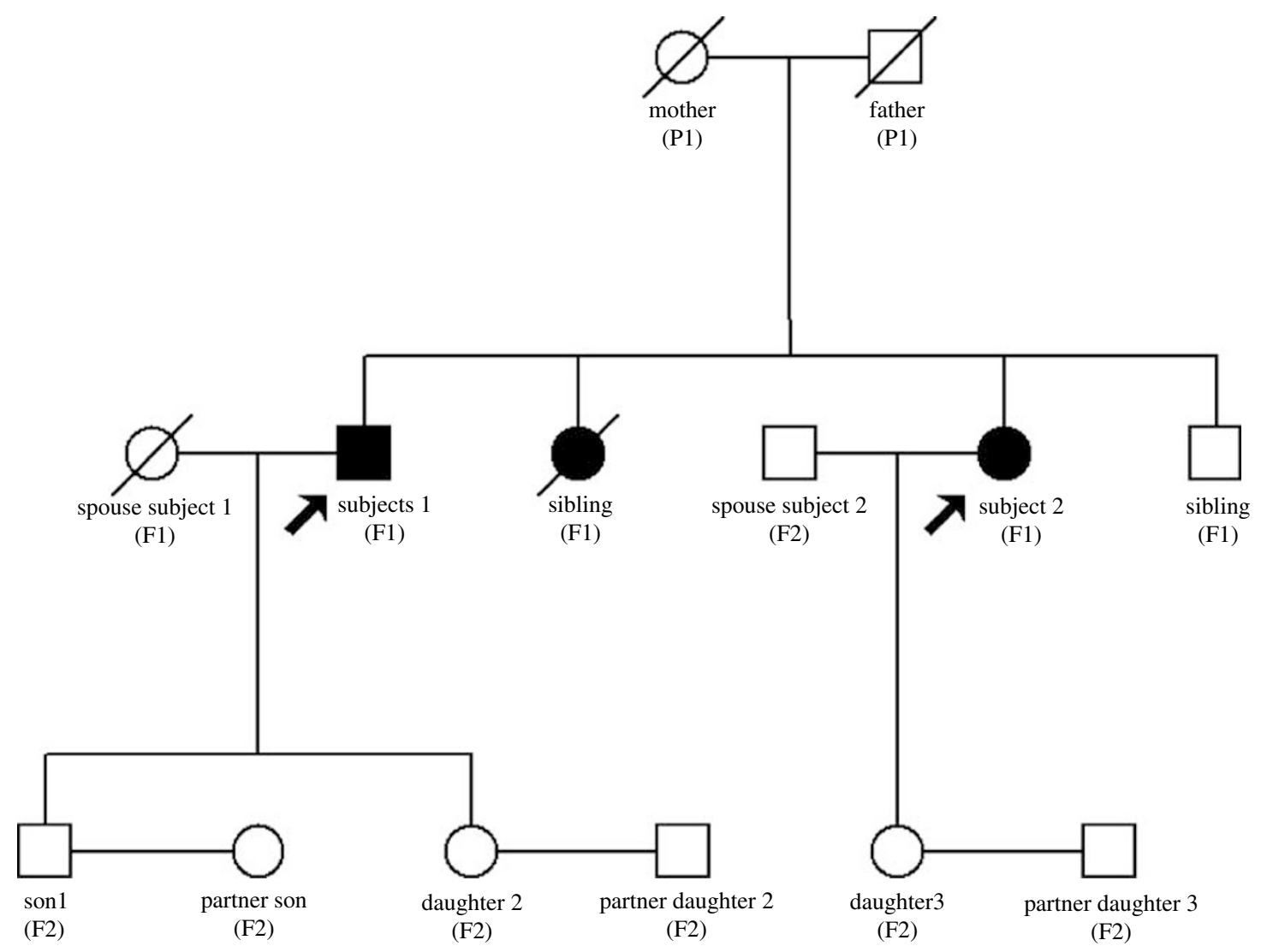

Figure 1 Representative pedigree. The closed symbols represent the long-lived individuals; the arrows point at the ascertained long-living subjects. P1, F1 and F2 represent the three generations included in the study.

with the general population using standardised mortality ratios (SMRs). The SMR is the ratio of the observed number of deaths in the study relative to the expected number of deaths in the general population, adjusted for sex and calendar period. A description of the method has been described by others. ${ }^{16,17}$ Second, the mortality between different groups was directly compared using Cox regression analysis. Level of significance was set at 0.05 .

\section{Results}

Recruitment to the Leiden Longevity Study started in July 2002. The number and age distribution of all family members of the first 100 families included in the study are listed in Table 1 . The characteristics described are derived from generations P1, F1, and F2. The proportion of deceased subjects increases with an earlier year of birth $(\mathrm{P} 1 \rightarrow \mathrm{F} 1 \rightarrow \mathrm{F} 2)$. The proportion of deceased males in generations F1 and F2 is higher than the proportion of deceased females. There were two living male spouses of long-living females, while there were 21 living female spouses of long-living males.
Table 1 Characteristics of the first 100 families

\begin{tabular}{|c|c|c|c|c|}
\hline & & Males & & emales \\
\hline & $n$ & Age & $n$ & Age \\
\hline Parents of lor & iving s & jects (P1) & & \\
\hline Deceased & 100 & $78(68-87)$ & $98^{\mathrm{a}}$ & $80(67-88)$ \\
\hline Total sibships & $F 1)$ & & & \\
\hline Alive & 106 & $90(84-93)$ & 228 & $92(89-95)$ \\
\hline Deceased & 238 & $75(47-84)$ & 152 & $84(61-90)$ \\
\hline Spouses of lo & living & bjects ${ }^{\mathrm{c}}(F 1)$ & & \\
\hline Alive & 2 & $88(86-89)$ & 21 & $86(83-89)$ \\
\hline Deceased & 119 & $76(68-84)$ & 36 & $81(78-86)$ \\
\hline Offspring of I & -living & ubjects (F2) & & \\
\hline Alive & 351 & $59(54-64)$ & 371 & $59(55-65)$ \\
\hline Deceased & 51 & $50(26-59)$ & 31 & $33(6-56)$ \\
\hline Partners of $\mathrm{c}$ & $n g$ of & ng-living sub & $(F 2)$ & \\
\hline Alive & 93 & $62(57-66)$ & 101 & $57(52-61)$ \\
\hline
\end{tabular}

Age displayed as median (interquartile range). P1, F1 and F2 represent the three generations included in the study (see also Figure 1).

${ }^{\text {a }}$ Two mothers had unknown dates of birth.

${ }^{\mathrm{b}}$ Total sibship includes the long-lived subjects and all the siblings.

${ }^{c}$ Calculations include only the first spouse.

${ }^{\mathrm{d} C}$ Calculations include only the partners who participated in the study. 
Table 2 Mortality of the parents, siblings, offspring and partners of the included sib pairs compared with the general Dutch population

\begin{tabular}{|c|c|c|c|c|c|c|}
\hline & \multirow[b]{2}{*}{$n$} & & \multicolumn{3}{|c|}{ Standardised mortality ratio $(95 \% \mathrm{Cl})$} \\
\hline & & $\begin{array}{c}\text { Observed } \\
\text { deaths }\end{array}$ & $\begin{array}{c}\text { Expected } \\
\text { deaths }\end{array}$ & All & Males & Females \\
\hline \multicolumn{7}{|l|}{ Total sibship (F1) } \\
\hline Including all participating long-living subjects & 724 & 390 & 1194 & $0.31(0.28-0.34)$ & $0.43(0.38-0.49)$ & $0.24(0.20-0.28)$ \\
\hline Excluding all participating long-living subjects & 503 & 390 & 591 & $0.66(0.60-0.73)$ & $0.71(0.63-0.81)$ & $0.59(0.50-0.69)$ \\
\hline Partners of long-living subjects ${ }^{c}$ (F1) & 178 & 155 & 161 & $0.95(0.82-1.12)$ & $1.00(0.84-1.20)$ & $0.82(0.59-1.14)$ \\
\hline
\end{tabular}

P1, F1 and F2 represent the three generations included in the study (see also Figure 1).

a Person years were counted from the date of birth of the youngest long-living subject.

${ }^{\mathrm{b}}$ Two parents had unknown dates of birth.

Includes only the first spouse of long-living subjects; person years were counted from the date of marriage.

The median MMSE score of the long-living subjects, indicating global cognitive functioning, was 25 (interquartile range (IQR) 21-27). Severe cognitive impairment (MMSE <19) was found in 14\% of the long-living subjects. The median Barthel and Fillenbaum scores, measuring disability, were 18 (IQR 15-20) and 8 (IQR $4-11$ ). Of the long-living subjects, $26 \%$ had a Barthel score of 20, indicating they are competent to perform all ADL independently. The proportion of participants who were able to perform all seven items of the Fillenbaum independently was $5 \%$. The median score on the Cantril ladder, a test to measure well-being, was 8 points out of 10 (IQR 7-8).

Table 2 lists the mortality characteristics of the various generations in the included families, expressed as SMR. An SMR below unity indicates that the mortality in the included sample is lower than in the general population, adjusted for sex, age distribution, and calendar time. The SMR for all 221 long-living participants and their 503 siblings was 0.31 (95\% CI $0.28-0.34$ ). When we excluded the 221 long-living participants, the SMR was 0.66 (95\% CI $0.60-0.73)$ for the 503 remaining brothers and sisters. The survival benefit is also present in the parents $(\mathrm{SMR}=0.76,95 \%$ CI $0.66-0.87)$ and in the offspring of the long-living participants $(\mathrm{SMR}=0.65$, 95\% CI 0.51-0.80). Moreover, the spouses of the long-living participants, who have a similar socioeconomic background as the long-living participants themselves, have a mortality pattern similar to that of the general population (SMR 0.95, 95\% CI $0.82-1.12)$.

In a direct comparison, the relative mortality risk of the brothers and sisters of the long-living participants versus the spouses of the long-living participants was 0.65 (sex-adjusted hazard ratio $0.65,95 \%$ CI $0.54-0.80$ ). The relative mortality risk between the siblings and the spouses was $0.66(95 \%$ CI $0.52-0.83)$ for the men and 0.65 (95\% CI $0.44-0.94)$ for the women.

\section{Discussion}

Using the first 100 families of the Leiden Longevity Study, we found a consistent survival benefit in the parents, the siblings, and the offspring of the long-living sib pairs. This clearly indicates that we have succeeded in generating a study sample that is enriched for longevity. As the benefit was present in all family members of the long-living sib pairs but not in their partners, we think it is likely that the survival benefit is not attributable to ascertainment bias or to environmental factors but is caused by heritable factors.

We have two arguments to infer that the survival benefit is caused by heritable factors. First, there is a clear survival advantage in all three generations of the included families. The parents (P1), siblings (F1), and offspring (F2) of the included long-living subjects all have a comparable survival benefit. The fact that family members beyond the participating long-living sib pairs have a lower mortality risk makes ascertainment bias unlikely. Second, in line with expectation, a direct comparison of the survival of the siblings of the long-living participants with the spouses of the long-living participants showed a survival benefit of 0.65 . We think that the adult environmental conditions of the siblings of the long-living participants are likely to be similar to the adult environmental conditions of the spouses of the long-living participants. Moreover, although it is possible that the early life conditions of the two groups are different, we consider it unlikely that familial nongenetic preadult environmental factors explain the observed survival benefit of 0.65 .

In comparison with the general Dutch population, we found a small, nonsignificant survival benefit of the 
partners of the long-living subjects. As high socio-economic background is associated with higher life expectancy, ${ }^{18}$ our sample might have a small overrepresentation of families with higher socio-economic background. Hence, we think that the small, nonsignificant survival benefit of the partners of the long-living subjects is likely to be caused by environmental factors. Moreover, we could not calculate an SMR of the partners of the offspring of the long-living participants because we only included living partners. Therefore, any comparison with the general population would have revealed a survival benefit, which is clearly caused by this selective inclusion.

The cognitive function of the long-living subjects is comparable with other studies of octo- and nonagenarians. ${ }^{19}$ According to expectation, the number of subjects who could independently do all ADL was higher than the number of subjects who could perform all IADL independently. ${ }^{20}$ A median score of 8.0 for well-being is in the same range as the elderly of 85 years and older. ${ }^{21}$ The scores on these tests indicate that our study sample is a good representation of the general population of the oldest of the Dutch elderly. Moreover, as the aim of our study is to locate genetic loci explaining the interindividual differences in human longevity, we decided not to collect any environmental risk factors associated with age-related diseases or longevity. For, so far as an unintended selection of a beneficial environment can explain for the survival benefit, we emphasise that mortality in the offspring was $35 \%$ lower than expected whereas it was not different from the general population in the spouses. These data strongly suggest that the survival benefit is due to familial, genetic factors.

Perls et $a l^{22}$ demonstrated that siblings of centenarians have a four-fold increased probability of surviving to the age of 91 years $\left(\lambda_{\mathrm{s}}=4\right)$. Kerber et $\mathrm{al}^{23}$ calculated that siblings of subjects achieving the 97 th percentile of excess longevity (for males this corresponded with an age of 95 years and for women an age of 97 years) had a $\lambda_{\mathrm{s}}$ of 2.30 to achieve the 97 th percentile. Normally, $\lambda_{s}$ is the probability for a long-living individual (not a sib pair) to have a longliving sibling divided by the probability of this in the general population. However, our study design does not permit this calculation as we included sib pairs in our study.

An important strength of our study is that the majority of analyses were carried out in subjects after exclusion of the long-living sib pairs. The parents, brothers and sisters, and offspring of the long-living participants all had an extended survival of about 0.70 . The analysis where the sib pairs were included showed a survival benefit of 0.31 . However, this is an overestimation of the survival benefit because of ascertainment bias since it also includes the long-living subjects. Hence, the true genetic enrichment of the included long-living sib pairs is probably between these two values. Another important strength is that all data were verified by official documents and population-based registries.

In conclusion, we observed familial clustering of extended survival in three generations in families included in the Leiden Longevity Study. In this study design of nonagenarian subjects, we distinguished shared environmental influences and a significant genetic component contributing to extended survival. Mapping of longevity genes in this study allows for future linkage analysis. The two generation inclusion further enables a combination of linkage and association analysis.

\section{References}

1 Finch CE: Longevity, Senescence, and the Genome. Chicago: The University of Chicago Press, 1990.

2 Zwaan BJ: The evolutionary genetics of ageing and longevity. Heredity 1999; 82: 589-597.

3 Lakowski B, Hekimi S: Determination of life-span in Caenorhabditis elegans by four clock genes. Science 1996; 272: 1010-1013.

4 Migliaccio E, Giorgio M, Mele S et al: The p66shc adaptor protein controls oxidative stress response and life span in mammals. Nature 1999; 402: 309-313.

5 Miller RA, Chrisp C, Jackson AU, Galecki AT, Burke DT: Coordinated genetic control of neoplastic and nonneoplastic diseases in mice. J Gerontol A Biol Sci Med Sci 2002; 57: B3-B8.

6 Herskind AM, McGue M, Holm NV, Sorensen TI, Harvald B, Vaupel JW: The heritability of human longevity: a populationbased study of 2872 Danish twin pairs born 1870-1900. Hum Genet 1996; 97: 319-323.

7 Iachine IA, Holm NV, Harris JR et al: How heritable is individual susceptibility to death? The results of an analysis of survival data on Danish, Swedish and Finnish twins. Twin Res 1998; 1: 196-205.

8 Perls TT, Wilmoth J, Levenson R et al: Life-long sustained mortality advantage of siblings of centenarians. Proc Natl Acad Sci USA 2002; 99: 8442-8447.

9 Skytthe A, Pedersen NL, Kaprio J et al: Longevity studies in GenomEUtwin. Twin Res 2003; 6: 448-454.

10 Puca AA, Daly MJ, Brewster SJ et al: A genome-wide scan for linkage to human exceptional longevity identifies a locus on chromosome 4. Proc Natl Acad Sci USA 2001; 98: $10505-10508$.

11 Reed T, Dick DM, Uniacke SK, Foroud T, Nichols WC: Genomewide scan for a healthy aging phenotype provides support for a locus near D4S1564 promoting healthy aging. J Gerontol A Biol Sci Med Sci 2004; 59: 227-232.

12 Folstein MF, Folstein SE, McHugh PR: 'Mini Mental State': a practical method for grading the cognitive state of patients for the clinician. J Psychiatr Res 1975; 12: 189-198.

13 Mahoney FI, Barthel DW: Functional evaluation: the Barthel index. Md State Med J 1965; 14: 61-65.

14 Fillenbaum GG: Screening the elderly. A brief instrumental activities of daily living measure. J Am Geriatr Soc 1985; 33: $698-706$.

15 Cantril H: The Pattern of Human Concern. New Brunswick, NJ: Rutgers University Press, 1965.

16 Hille ET, Westendorp RGJ, Vandenbroucke JP, Rosendaal FR: Mortality and causes of death in families with the factor V Leiden mutation (resistance to activated protein C). Blood 1997; 89: $1963-1967$.

17 Rosendaal FR, Heijboer H, Briët E et al: Mortality in hereditary antithrombin - III deficiency - 1830-1989. Lancet 1991; 337: $260-262$. 
18 Vagero D, Leon D: Effect of social class in childhood and adulthood on adult mortality. Lancet 1994; 343: 1224-1225.

19 Heeren TJ, Lagaay AM, von Beek WC, Rooymans HG, Hijmans W: Reference values for the Mini-Mental State Examination (MMSE) in octo- and nonagenarians. J Am Geriatr Soc 1990; 38: 1093-1096.

20 Bootsma-van der Wiel A, Gussekloo J, de Craen AJ et al: Disability in the oldest old: 'can do' or 'do do'? I Am Geriatr Soc 2001; 49: 909-914.
21 von Faber M, Bootsma-van der Wiel A, van Exel E et al: Successful aging in the oldest old: who can be characterized as successfully aged? Arch Intern Med 2001; 161: 2694-2700.

22 Perls TT, Bubrick E, Wager CG, Vijg J, Kruglyak L: Siblings of centenarians live longer. Lancet 1998; 351: 1560.

23 Kerber RA, O'Brien E, Smith KR, Cawthon RM: Familial excess longevity in Utah genealogies. J Gerontol A Biol Sci Med Sci 2001; 56: B130-B139. 